\title{
Dynamic Antenna Alignment Control in Microwave Air-Bridging for Sky-Net Mobile Communication Using Unmanned Flying Platform
}

\author{
Chin E. Lin and Ying-Chi Huang \\ Department of Aeronautics and Astronautics, National Cheng Kung University, Tainan, Taiwan \\ Correspondence should be addressed to Chin E. Lin; chinelin@mail.ncku.edu.tw
}

Received 14 October 2014; Accepted 8 March 2015

Academic Editor: Sing Kiong Nguang

Copyright (C) 2015 C. E. Lin and Y.-C. Huang. This is an open access article distributed under the Creative Commons Attribution License, which permits unrestricted use, distribution, and reproduction in any medium, provided the original work is properly cited.

\begin{abstract}
This paper presents a preliminary study on establishing a mobile point-to-point (P2P) microwave air-bridging (MAB) between Unmanned Low Altitude Flying Platform (ULAFP) and backhaul telecommunication network. The proposed Sky-Net system relays telecom signal for general mobile cellphone users via ULAFP when natural disaster sweeps off Base Transceiver Stations (BTSs). Unlike the conventional fix point microwave bridging application, the ULAFP is cruising on a predefined mission flight path to cover a wider range of service. The difficulty and challenge fall on how to maintain antenna alignment accurately in order to provide the signal strength for MAB. A dual-axis rotation mechanism with embedded controller is designed and implemented on airborne and ground units for stabilizing airborne antenna and tracking the moving ULAFP. The MAB link is established in flight tests using the proposed antenna stabilizing/tracking mechanism with correlated control method. The result supports backbone technique of the Sky-Net mobile communication and verifies the feasibility of airborne e-Cell BTS.
\end{abstract}

\section{Introduction}

Ever since 1990s, mobile communication had been gradually becoming the most dependent livelihood communication means on handy sets. Base Transceiver Stations (BTSs) for mobile communications are usually built on the ground for services. On the natural disasters, such as earthquake, typhoon, or flood, the BTSs as well as power systems are easily affected or destroyed and thus black out communications from outside forces to inside victims. The most important part for disaster rescue would be the recovery of communications. Any common methods to establish mobile communication for any users within the disaster area would be most appreciated even just for a short period.

The Sky-Net project addresses the establishment and recovery of a temporary mobile phone service for general users by using Unmanned Low Altitude Flying Platform (ULAFP) carrying with an airborne e-Cell BTS to relay mobile signal to distant ground BTS. The proposed Sky-Net connects mobile communication into backbone network of Chunghwa Telecom Company in Taiwan.

The concept of relaying wireless signal via flying platform had been studied in the past decade. Literatures categorized different flying platforms from high or low flight altitudes. Widiawan and Tafazolli [1] introduced the necessary part of the wireless communication relay using High-Altitude Platform (HAP), including platform type, energy consumption for long time mission, and communication loading for the system. Because of the Line-of-Sight (LoS) characteristics of the flying platform, this kind of airborne relay equipment can provide more coverage or increase the link capacity for heavy loading area. The coverage emulation studied by Feng et al. [2] also adopted multi-HAP on signal relay missions. The increment of link capacity was proved by Grace et al. [3] and Elshaikh et al. [4]. The airborne relay equipment uses various wireless link types. Because of the wide usage and application of personal electronic devices, like mobile phone or laptop, relaying wireless signal with Internet service 


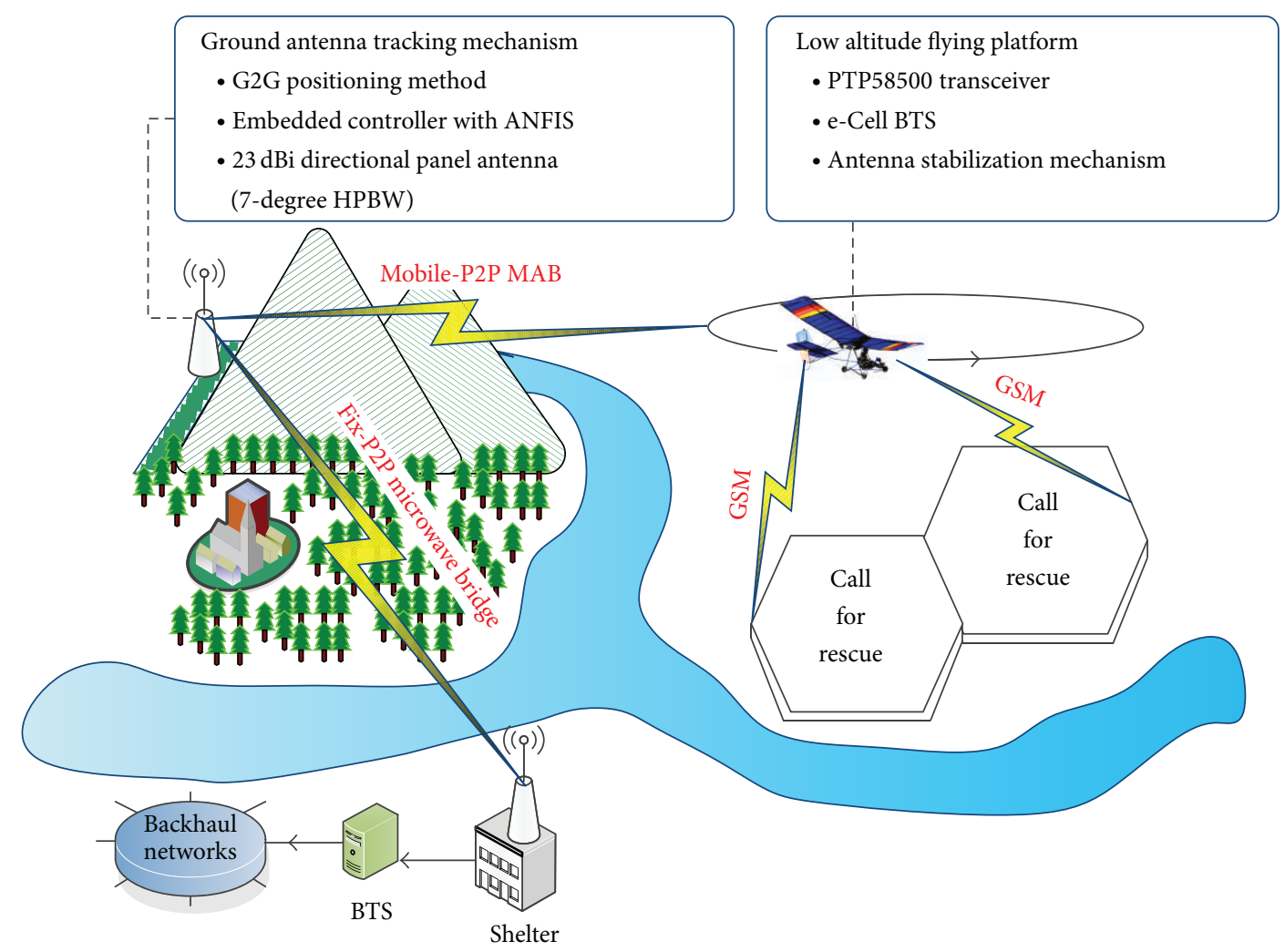

FIGURE 1: The proposed Sky-Net mobile signal relaying concept.

becomes the major issue on such research. Hariyanto et al. [5] introduced a study on Emergency Broadband Access Network (EBAN) application using balloon to hover at altitude range from 100 to 500 meters. Hariyanto sorted out three major alternate solutions for wireless Internet service, such as WiMAX, 3G, and WiFi. Qiantori et al. [6] demonstrated the result of airborne WiFi service using two balloons to carry transceiver module. They work on launching a balloon hovering at $440 \mathrm{~m}$ (AGL) to provide service area up to $3.88 \mathrm{~km}$. The maximum throughput of this system is limited under $0.8 \mathrm{Mbps}$, which is just enough for transmitting voice data for user [6]. The other hybrid type of relaying is introduced by Guo et al. [7] to propose a WiFi link between users and the drone. The latter part of airborne decode-and-forward (DaF) device exchanges data pack between the drone and the nearest BTS. However, in the Sky-Net project, system architecture is proposed as directly relayed signal into backbone networks of Telecom Company, which is operating under E1 protocol in this paper. Besides, instead of using balloon as LAFP, the Sky-Net proposed a fix-wing unmanned ultralight aircraft as flying platform like HeliNet project in Europe [8] in order to fly against the unpredictable weather conditions. Under Sky-Net concept, mobile signal is carried by microwave with frequency at $5.8 \mathrm{GHz}$ in order to achieve high bandwidth for steady link. The relay mechanism can be sorted into two parts as Fix-P2P and Mobile-P2P, separately. Like general microwave bridging, Fix-P2P relays signal from functional BTS to ground antenna tracking station for Mobile-P2P, as shown in Figure 1.
In order to establish a microwave air-bridging (MAB), a directional antenna is adopted with advantage on highly narrowed power beam. It can also support farther wireless link establishment and higher link quality. Yet, the narrow antenna pattern comes with directional restriction; once the flying platform is out of the coverage area of the ground directional antenna, signal strength would drop and therefore affect the integrity of MAB. On the other hand, if the flying platform would meet inevitable attitude change during cruise flight, the polarization or beam direction of airborne antenna is changing in whole flight time, which may affect wireless link quality too.

For the ULAFP and ground unit, a dual-axis rotation platform is proposed in this paper in order to provide well directional antenna alignment from the flying platform and maintain steady attitude for airborne gimbal on mounting antenna. Both ground and airborne rotation mechanisms have to apply proper control method to achieve the required $\mathrm{MAB}$ performance. Flight tests for verifying the performance and integrity of the proposed $\mathrm{MAB}$ for mobile signal relaying operation are demonstrated. It supports the evidence for the reliable backbone technique for further developments and realizations of the Sky-Net project.

\section{Antenna Stabilization/Tracking Mechanism}

In consideration to maintain the most antenna pattern overlap ratio for wireless link, airborne antenna needs to be 
covered under the coverage area of the ground directional antenna, and vice versa. Smart antenna with beam forming performance is an ideal solution for such condition; Falletti et al. [9] did the analysis of Bit Error Rate (BER) for bot high speed train and HAP antenna. Falletti verified that the smart antenna showed suitable wireless relaying to mobile target. On the other hand, several antenna types for relaying different carrier frequency were studied by Miura and Suzuki [10]. Both electrical and mechanical scanning techniques are compared. However, by reducing system complexity, the Sky-Net project proposes a dual-axis rotation mechanism on both airborne and ground units as directly connected with backbone networks in mobile telecommunication. While the purpose ensures the coherence of polarization of airborne antenna during the flight mission, the airborne dual-axis mechanism needs to compensate the dynamic changes during flights. The ULAFP attitude in roll and pitch motion varies due to flight control or aerodynamic disturbance. Consequently, it is most required to control the ground antenna tracking unit to fit its performance on aiming at the ULAFP in Sky-Net operation. Both rotation mechanisms have correlated control algorithm and mechanism design, separately, for microwave alignment.

2.1. Airborne Antenna Stabilization. In the Sky-Net project, a $10 \mathrm{dBi}$ omnidirectional linearly polarized antenna is selected to match with the other $2.5 \mathrm{dBi}$ circularly polarized antenna to fit the multiple-input-multiple-output (MIMO) performance for Motorola PTP58500 transceiver, which is telecommunication signal relaying equipment for long range Ethernet bridging. The major task of airborne mechanism should maintain the antenna's polarization for ULAFP during cruise flight. In other words, the antenna in vertical polarization should be installed to point to the ground regardless of ULAFP's attitude and flight performance. This kind of gimbal has been widely studied for applications to aerial photography from airborne systems, such as fix-wing or multirotor Unmanned Aerial Vehicle (UAV) [11]. Figure 2 illustrates the dimension and coordinates arrangement of the proposed antenna stabilization mechanism.

Using the microelectromechanical system (MEMS) technique, the Inertial Measurement Unit (IMU) now could be small enough to merge into microcontroller inside a small piece of Printed Circuit Board (PCB). It is therefore widely used on embedded control applications in robotics. UAV and the proposed stabilization mechanism are typical uses. Studies have been made for attitude estimation to control the gimbal mechanism [12]. Madgwick et al. [13] introduced the real-time attitude estimation in microcontroller with algorithm as less calculation cost as possible in dedicate realization. In this paper, Madgwick et al's method is adopted as attitude information for controlling the gimbal with modifications. The proposed stabilization block diagram is shown in Figure 3.

In Figure 3, the control core in each control loop solves the command to manipulate the mechanism attitude. Either pitch angle or roll angle in the gimbal should be maintained under designed thresholds. Otherwise the control law will

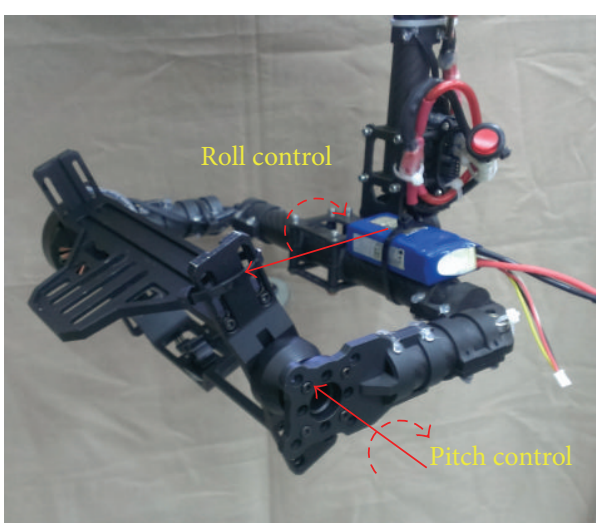

FIgURE 2: Airborne dual-axis antenna stabilization gimbal.

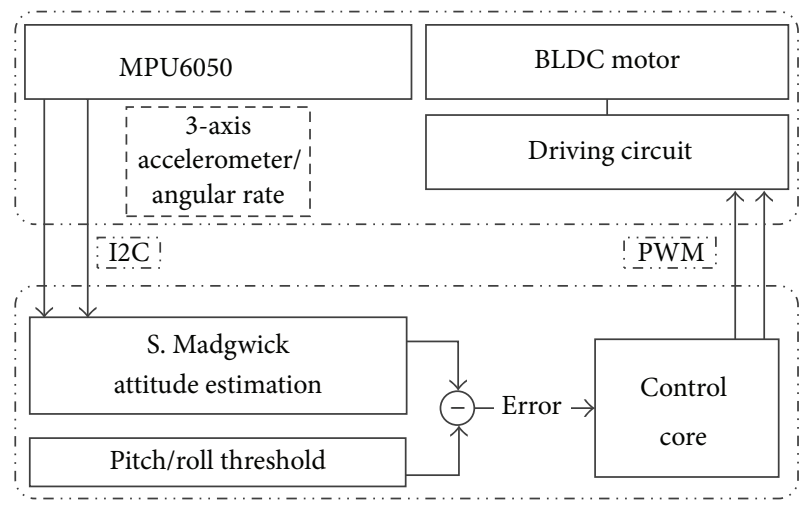

FIGURE 3: Block diagram of antenna stabilization control.

output the driving signal for Brushless DC Motor (BLDC) in sequent of roll and pitch axes to compensate the angle offset due to ULAFP motion or external disturbance. In the flight tests to verify the stabilization performance of designed gimbal, the data are logged as roll and pitch angles of the gimbal referring to ULAFP attitude, correspondingly, as shown in Figure 4.

Blue line in Figure 4 represents the attitude changing status of ULAFP during the flight stage. The glitch on blue line data reveals the inevitable vibration when the antenna mechanism is directly mounted onto ULAFP. It may cause the antenna polarization offset and lead MAB throughput to drop down temporarily or even lose connection. The proposed gimbal stabilization mechanism should be effectively maintained for both pitch and roll angles in good alignment to around zero degrees while ULAFP is flying. This is shown by the red line in Figure 4.

2.2. Ground Antenna Tracking Mechanism. In addition to airborne stabilization mechanism, the ground tracking unit mounting with highly directional panel antenna needs even more accurate positioning control on elevation and azimuth angle for tracking the ULAFP. The ground antenna tracking mechanism should cover the airborne antenna under HalfPower Beam Width (HPBW) area. The ground antenna tracking mechanism is driven by stepper motor with gear 

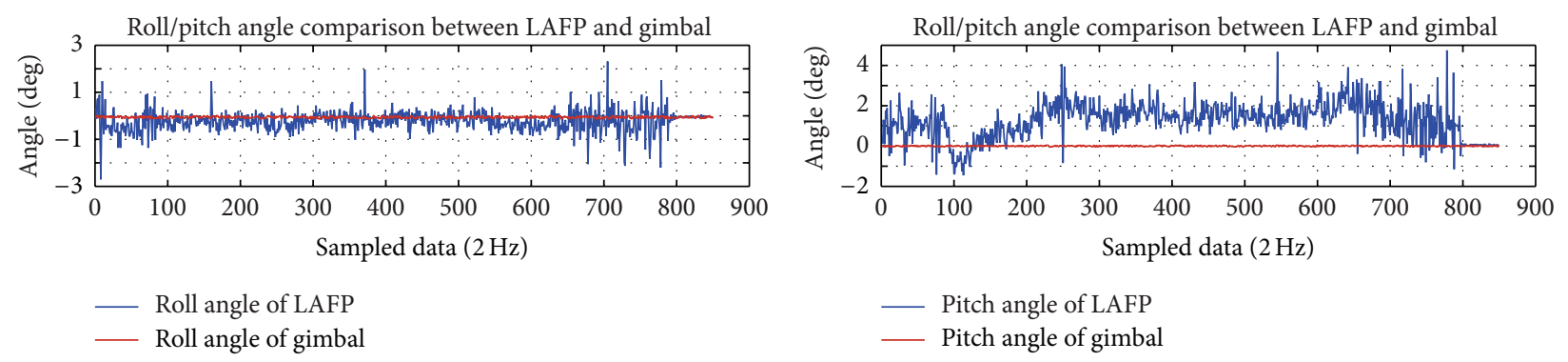

FIgURE 4: Gimbal roll and pitch angles referring to ULAFP attitude.

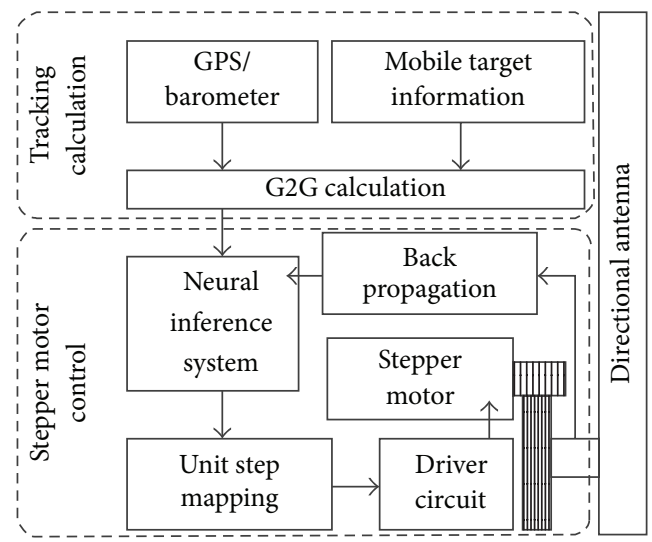

FIGURE 5: Block diagram of ground antenna tracking unit.

mechanism. Therefore, the tracking mechanism can be categorized into tracking calculation and stepper motor control, as shown in Figure 5.

For applications like target tracking out of visual range, the related angle displacement between the ground tracking unit and the ULAFP is small. The tracking mechanism needs very high accuracy feature in perturbation control much more than its rotation speed. And also, as the Line-ofSight (LoS) distance between the ULAFP and the ground tracking unit is long enough, GPS error becomes negligible in calculation. A simple but efficient tracking method based on GPS-to-GPS (G2G) method is adopted in the Sky-Net project. By using GPS and barometric data, the related azimuth and elevation angles can be solved easily. However, as distance increases, the calculation error appears significantly because of the earth curvature, especially on the elevation angle. G2G method assumes that the ground tracking unit and the ULAFP are on the same surface of coordinates. Both sides are located in the same Cartesian Coordinates system. Figure 6 indicated the error accumulation as distance $S_{1}$ increases.

If the ground tracking unit and the ULAFP are close enough, angle calculation can be simplified into 3-axis Cartesian Coordinates directly. By transforming the GPS position into another coordinates system, such as TM2 in TWD97, distance between the ground antenna tracking unit and the ULAFP can be obtained, marked as $S_{1}$ in Figure 6. In addition to coordinate transformation, the altitude difference can be

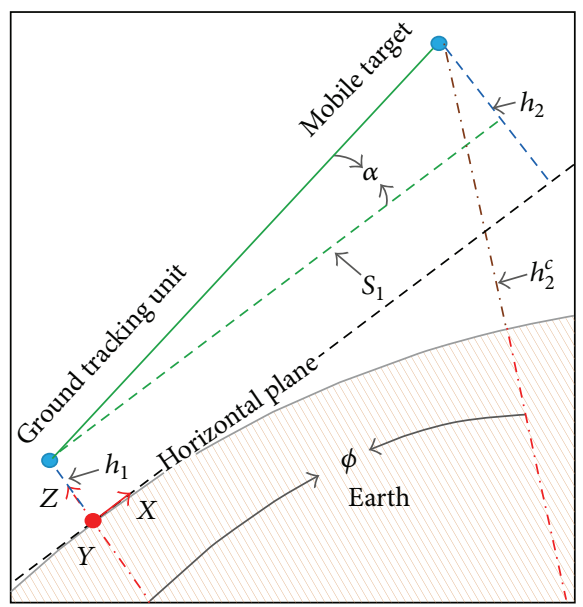

FIGURE 6: Elevation angle error due to earth curvature.

calculated from barometric data and GPS altitude data. The elevation angle $\alpha$ can be expressed as

$$
\alpha=\arctan \left(\frac{h_{2}-h_{1}}{S_{1}}\right) .
$$

However, as $S_{1}$ is far from both terminals, the curvature of the earth will evidently affect the accuracy of elevation angel $\alpha$. Figure 6 also shows how the curvature makes the error bigger as $S_{1}$ increases. In TWD97 data, the earth model is an ellipsoid and its radius scale is relatively huge compared to ULAFP flight path. Within a small region, the earth is reasonably assumed as a sphere not an ellipsoid for simplicity. Therefore between the ground and the airborne terminals in Figure 6, the earth radius is $R$ and the inclination angle between them toward earth center is $\phi$; the elevation angle $\alpha$ of the ground terminal can be recalculated as

$$
\begin{gathered}
d_{1}=R^{2}+\left(h_{2}^{c}+R\right)^{2}-2 R\left(h_{2}^{c}+R\right) \phi, \\
d_{2}=\left(h_{1}+R\right)^{2}+\left(h_{2}^{c}+R\right)^{2}-2\left(h_{1}+R\right)\left(h_{2}^{c}+R\right) \cos \phi, \\
\alpha=\cos ^{-1}\left(\frac{h_{1}^{2}+d_{1}^{2}-d_{2}^{2}}{2-h_{1} d_{1}}\right) .
\end{gathered}
$$

The relative azimuth and elevation angles obtained from G2G calculation output control parameters for stepper motor 
TABLE 1: Step division of stepper motor (full-step@1.8 $8^{\circ}$.

\begin{tabular}{lccc}
\hline Mode & Resolution & Mode & Resolution \\
\hline 1 & $1 / 1$ & 9 & $1 / 40$ \\
2 & $1 / 2$ & 10 & $1 / 50$ \\
3 & $1 / 2.5$ & 11 & $1 / 100$ \\
4 & $1 / 5$ & 12 & $1 / 125$ \\
5 & $1 / 8$ & 13 & $1 / 200$ \\
6 & $1 / 10$ & 14 & $1 / 250$ \\
7 & $1 / 20$ & 15 & $1 / 500$ \\
8 & $1 / 25$ & 16 & $1 / 1000$ \\
\hline
\end{tabular}

mechanism. Instead of direct driving stepper motor to the desired angle displacement, a neural inference system with back propagation updating mechanism is proposed in this paper. Its merit mainly shows the advantage unit step switching using stepper motor in the mechanism drive. Unit step division of the adopted stepper motor is shown in Table 1 . Combining with the division factor and its gear mechanism ration, the resolution of the ground antenna tracking unit hence becomes 0.18 degrees to 0.00018 degrees. It makes the antenna mechanism in microstep control.

As ULAFP flying along the designated mission path, cruising speed or actual path may be different due to the characteristic differences of flying vehicle, pilot, or even flight control computer. Hence, even though the flight path is redesigned and designated before taking off, the instant angle changing rate of elevation and azimuth axis is still different in the whole flight mission. The unit step switching feature of stepper motor is suitable in this implementation on angle positioning application with wide range angle displacement. Controller needs to be designed with capability on making appropriated unit step selection in order to consider both tracking accuracy and response speed. The proposed Adaptive Neural Fuzzy Inference System (ANFIS) plays the role of selecting the output unit step of stepper motor of each axis.

Figure 7 shows the typical 1st order Sugeno Fuzzy Model [14] adopted in this paper. Two input variables and one output system structure are proposed. Therefore, a MISO fuzzy logic can be expressed as the following human describing sentences:

$$
\begin{aligned}
& \text { Rule }_{1,1} \text { : If } x_{1} \text { is } A_{1} \text { AND } x_{2} \text { is } B_{1} \text { Then } y_{1,1} \text { is } C_{1,1} \\
& \text { Rule }_{1,2} \text { : If } x_{1} \text { is } A_{1} \text { AND } x_{2} \text { is } B_{2} \text { Then } y_{1,2} \text { is } C_{1,2} \text {. }
\end{aligned}
$$

The describing sentences use fuzzy linguistic label of membership function to express logic inference in human language, where $A=\left\{A_{1}, A_{2}, A_{3}, \ldots, A_{m}\right\}$ and $B=$ $\left\{B_{1}, B_{2}, B_{3}, \ldots, B_{n}\right\}$ are linguistic label set. Subscripts $m$ and $n$ of each fuzzy set vector represent the vector dimension, separately. As shown in Figure 7, the proposed ANFIS architecture is divided into four layers. Nodes in Figure 7 are defined as fix node for circle shape, which are predesigned and will not be changed in the whole inference process. On the other hand, the rectangle nodes are adaptive ones, which are the updating target for ANFIS in back propagation (BP) training.
The proposed neural fuzzy inference process can be classified into four layers: input, fire strength calculation, rule formation, and output, as below.

Layer 1 (Inputs). In layer one, two input variables are directly mapped into membership functions to get the corresponding membership grade:

$$
\begin{aligned}
& O_{1, i}^{1}=\mu_{A_{i}}\left(x_{1}\right), \quad i=1,2,3, \ldots, m, \\
& O_{2, j}^{1}=\mu_{B_{j}}\left(x_{2}\right), \quad j=1,2,3, \ldots, n .
\end{aligned}
$$

For arbitrary crisp input data, the $i$ th membership functions for input $x$ will be generated as $\mu_{A_{i}}(x)$ and $\mu_{B_{i}}(x)$. Superscript of $O^{e}$ represents the output of layer $e$.

Layer 2 (Fire Strength). Fire strength factor is defined as multiplication product of two input membership grades in order to represent the logical AND operation. This indicates the implementation strength of the corresponding rule. Therefore, in Layer 2, the output terms and the fire strength matrix $[\alpha]_{m, n}$ can be presented as

$$
\begin{gathered}
O_{i, j}^{2}=\mu_{A_{i}} \mu_{B_{j}} \\
{[\alpha]_{m, n}=\mu_{A}^{T} \cdot \mu_{B}=\left[\begin{array}{ccc}
\alpha_{1,1} & \cdots & \alpha_{1, n} \\
\vdots & \ddots & \vdots \\
\alpha_{m, 1} & \cdots & \alpha_{m, n}
\end{array}\right],}
\end{gathered}
$$

Layer 3 (Rule Formulation). In this layer, the core of Fuzzy Logic Controller (FLC) is shown as Rule Base Matrix (RBM). $\mathrm{RBM}$ is the decision maker to generate the appropriate output results. However, unless the designer is familiar with the control target, RBM could generate the region logical confliction or lack of system response. The Error Correction Mechanism (ECM) is set in this layer with the purpose of training and updating the value of each node until the output error scale converges into the desired margin. In this paper, a rule function is specially designed and set for generating the corresponding value of nodes in Layer 3, as shown below:

$$
O_{i, j}^{3}=f_{i, j}^{m, n}=(-m+2 i-1) p_{i, j}+(-n+2 j-1) q_{i, j},
$$

where $p_{i, j}$ and $q_{i, j}$ are the learning factor for nodes belonging to inputs $X_{1}$ and $X_{2}$, respectively. The rule function is designed for general stepper motor driven platform. The formulated rule values carry with $+/-$ signs to represent motor driving direction. According to the network in Figure 7, 


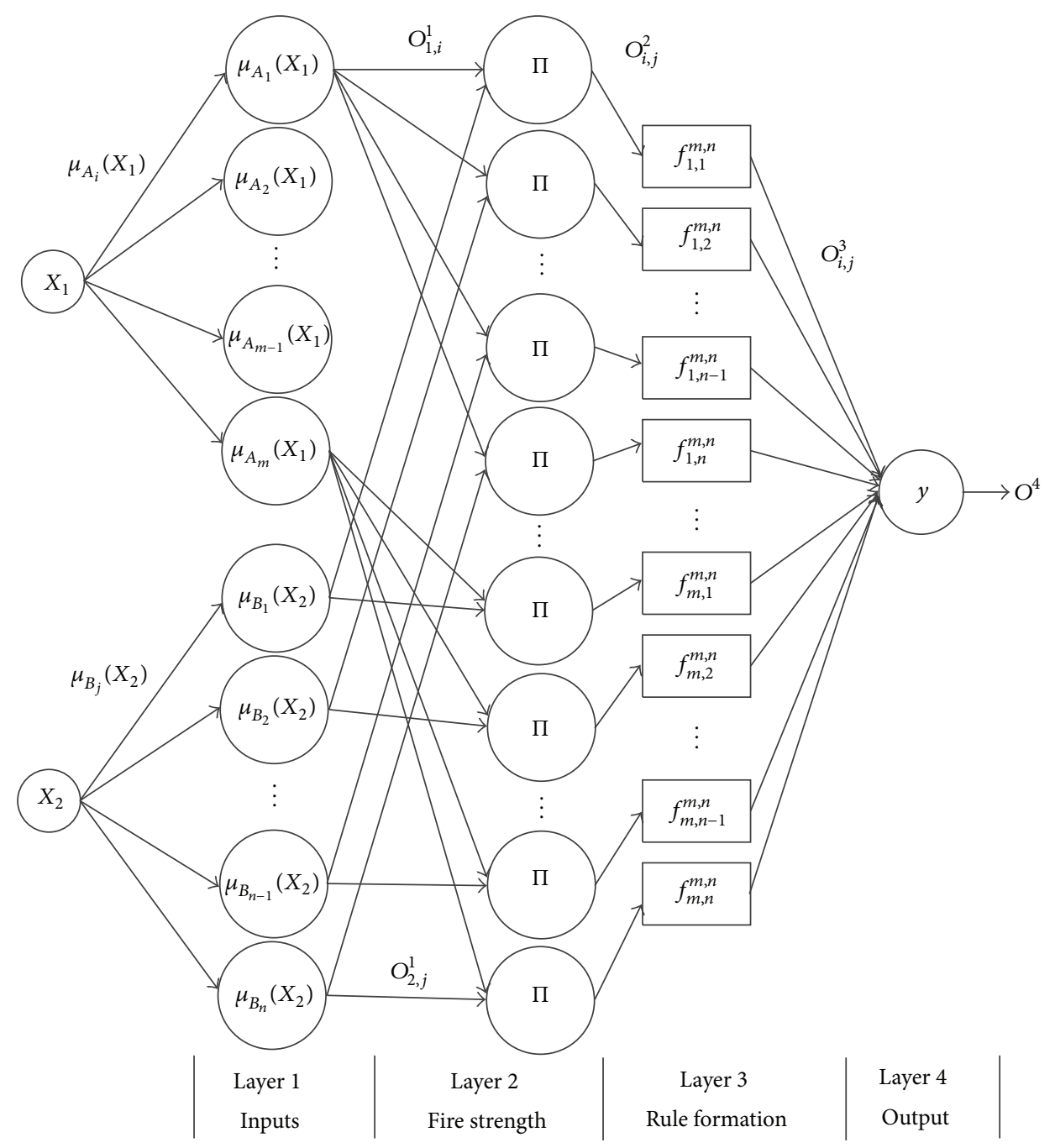

Figure 7: Architecture of adopted ANFIS.

output result from Layer 3 can be represented in matrix form as

$$
\begin{aligned}
{[\mathrm{RBM}]_{m, n} } & =[\alpha]_{m, n} \times\left[\begin{array}{ccc}
f_{1,1}^{m, n} & \cdots & f_{1, n}^{m, n} \\
\vdots & \ddots & \vdots \\
f_{m, 1}^{m, n} & \cdots & f_{m, n}^{m, n}
\end{array}\right] \\
& =\left[\begin{array}{ccc}
O_{1,1}^{3} & \cdots & O_{1, n}^{3} \\
\vdots & \ddots & \vdots \\
O_{m, 1}^{3} & \cdots & O_{m, n}^{3}
\end{array}\right] .
\end{aligned}
$$

Layer 4. Output result in Layer 4 is acquired using the gravity center method. The overall crisp output of fuzzy inference process is calculated in this single node. This is also called the defuzzification stage. Consider

$$
O^{4}=\frac{\sum_{i=1}^{m} \sum_{j}^{n} \alpha_{i, j} f_{i, j}^{m, n}}{\sum_{i=1}^{m} \sum_{j}^{n} \alpha_{i, j}} .
$$

In order to improve the result form of the neural fuzzy inference system, back propagation is adopted for adjusting rule formation parameters in Layer 3 of Figure 7. For a single output system, the goal of training reduces the value of the following cost function:

$$
E(k)=\frac{1}{2}(d(k)-y(k))^{2}=\frac{1}{2}(e(k))^{2} .
$$

At time $k, d(k)$ and $y(k)$ are the desired and actual system outputs, separately. The update law applied on arbitrary parameters $C_{i, j}$ in the neural network at time $k+1$ is the combination of its value at time $k$. The learning result and the momentum part are shown as

$$
\begin{gathered}
C_{i, j}(k+1)=C_{i, j}(k)-\eta^{c} \frac{\partial E(k)}{\partial C_{i, j}}+\tau^{c} \Delta C_{i, j}(k), \\
\frac{\partial E(k)}{\partial C_{i, j}}=-e(k) \frac{\partial O_{i, j}^{3}}{\partial C_{i, j}} .
\end{gathered}
$$


Learning rate and momentum factor are represented as $\eta^{c}$ and $\tau^{c}$, separately. Therefore, the update law of $p_{i, j}$ and $q_{i, j}$ of each node in Layer 3 at time $k$ can be expressed as

$$
\begin{aligned}
& p_{i, j}(k+1)=p_{i, j}(k)+\eta^{p} e(k) \frac{\partial O_{i, j}^{3}}{\partial P_{i, j}}+\tau^{p} \Delta p_{i, j}(k), \\
& q_{i, j}(k+1)=q_{i, j}(k)+\eta^{q} e(k) \frac{\partial O_{i, j}^{3}}{\partial q_{i, j}}+\tau^{q} \Delta q_{i, j}(k) .
\end{aligned}
$$

The partial differential parts can be expanded as follows:

$$
\begin{aligned}
& \frac{\partial O_{i, j}^{3}}{\partial P_{i, j}} \\
&= \frac{\partial}{\partial P_{i, j}} \\
& \quad \frac{\sum_{i=1}^{m} \sum_{j=1}^{n} \alpha_{i, j}\left\{(-m+2 i-1) p_{i, j}+(-n+2 j-1) q_{i, j}\right\}}{\sum_{i=1}^{m} \sum_{j}^{n} \alpha_{i, j}(k)} \\
&= \frac{\left(\partial / \partial P_{i, j}\right) \sum_{i=1}^{m} \sum_{j=1}^{n} \alpha_{i, j} f_{i, j}^{m, n}}{\alpha}(k) \\
& \bar{\alpha}(k)=\sum_{i=1}^{m} \sum_{j}^{n} \alpha_{i, j}(k)=\frac{\alpha_{i, j}(-m+2 i-1)}{\bar{\alpha}(k)} .
\end{aligned}
$$

Similarly, for $q_{i, j}$, the partial differential part in update law is

$$
\frac{\partial O_{i, j}^{3}}{\partial q_{i, j}}=\frac{\alpha_{i, j}(-n+2 j-1)}{\bar{\alpha}(k)} .
$$

According to the above equations, the change scale of BP process at time $k$ is determined by three factors: (a) the summation of fire strength of each of the membership functions in Layer 1, (b) labeled number of the corresponding nodes, and (c) the output error magnitude. The learning factor affects $O_{i, j}^{3}$ result directly. However, in the BP process, output error scale is possibly huge and leads to the overtraining result. It could cause the oscillation or divergence in training. A threshold is added into the BP before updating new value of target parameter in order to avoid the overtraining condition. The maximum value of each learning factor is determined according to the corresponding labeled value:

$$
\begin{gathered}
p_{i, j}^{\max }=\frac{O_{i, j_{\max }}^{3}}{(-m+2 i-1)}, \\
q_{i, j}^{\max }=\frac{O_{i, j_{\max }^{3}}^{3}}{(-n+2 j-1)} \\
O_{i, j_{\max }^{3}}^{3}=\operatorname{abs}(-m+1)+\operatorname{abs}(-n+1) .
\end{gathered}
$$

Therefore, the update law can be rewritten into the following equations:

$$
\begin{aligned}
& \bar{p}_{i, j}(k+1)=\min \left(p_{i, j}^{\max }, p_{i, j}(k+1)\right), \\
& \bar{q}_{i, j}(k+1)=\min \left(q_{i, j}^{\max }, q_{i, j}(k+1)\right) .
\end{aligned}
$$

In the beginning of the training process, the initial value of learning factor is set to 1 . Instead of using a random forming fuzzy surface, a rough established fuzzy surface is generated, and therefore the proposed method has ability to provide system with a degraded output, instead of leading system output with a conflict decision.

Verification of the proposed ANFIS control method for antenna tracking mechanism control is presented with flight test data in this study. Control core adopted by this paper is a microcontroller with Cortex-M4 architecture running at $180 \mathrm{MHz}$ as CPU speed. Figures 8 and 9 indicate the rule updating with the proposed rule formation method. Figures 10 and 11 show the difference on angle displacement error and online tracking result versus time between predesign and random set fuzzy surface under the proposed ANFIS method.

Both of predesigned and random set fuzzy surface training results are shown in Figures 8 and 9. Even though BP process can update the fuzzy surface at each calculation and makes the frequently used rule be well-trained, there are some decision confliction parts hidden by untrained blocks. On the other hand, the predesigned fuzzy surface has some regions that have not been trained in flight mission, too. Because of the logic coherence of fuzzy surface, the system can still generate suitable output decision and shorten training time compared with random set fuzzy surface method.

According to Figures 10 and 11, the tracking error reveals that the random set fuzzy surface has slower response on adapting a tracking mechanism. In tracking error chart, glitches from Figure 10 are the part that control core "step" into random set fuzzy surface part. Therefore, the potential logic confliction generates the inappropriate output for stepper motor. The tracking error is relatively larger than the proposed predesign fuzzy surface method, as shown in Figure 11. There is noticeable glitch difference on error chart in Figure 11 compared with Figure 10. Besides, because the tendency of fuzzy surface of the proposed method is coherent, it needs less number of training times for adjusting $p_{i, j}$ and $q_{i, j}$ on each node in neural networks. Under the above introduced antenna tracking accuracy, MAB establishment can be realized between ground antenna tracking mechanism and ULAPF.

\section{Verification of MAB Establishment}

In the flight test, an Eipper ultralight aircraft with license number JJ-2071 is converted in unmanned ultralight (UUL) aircraft using ULAFP for Sky-Net project $[15,16]$. In order to achieve 360-degree communication in the whole cruising path, two omnidirectional antennas with circular and linear polarization are installed on airborne antenna stabilization mechanism, as shown in Figure 12. 

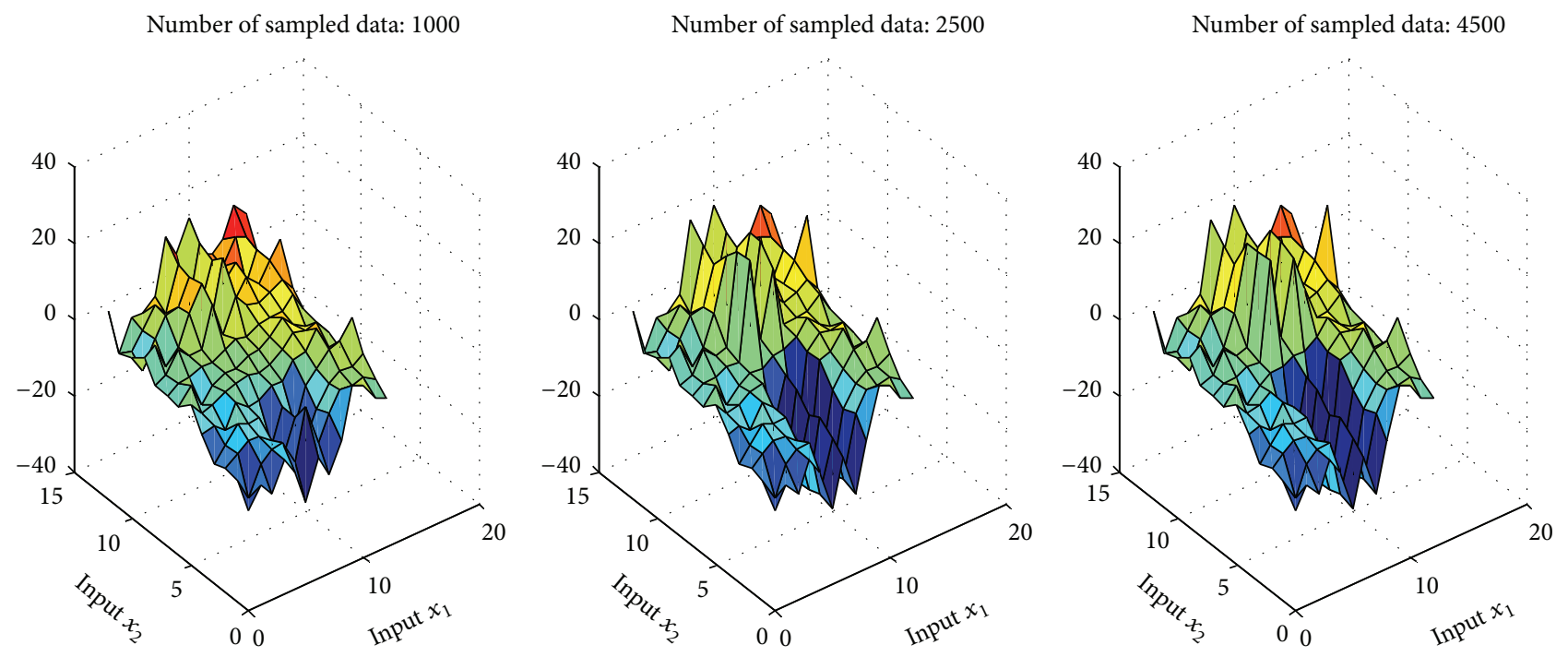

FIgURE 8: Training result of random set fuzzy surface.

Number of sampled data: 1000

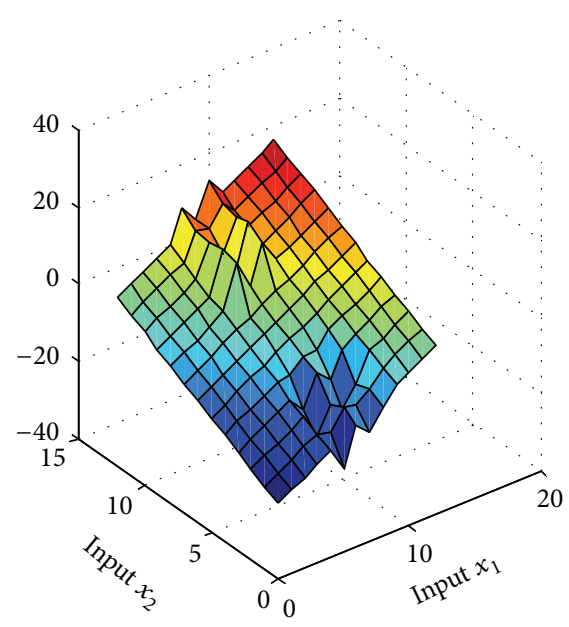

Number of sampled data: 2500

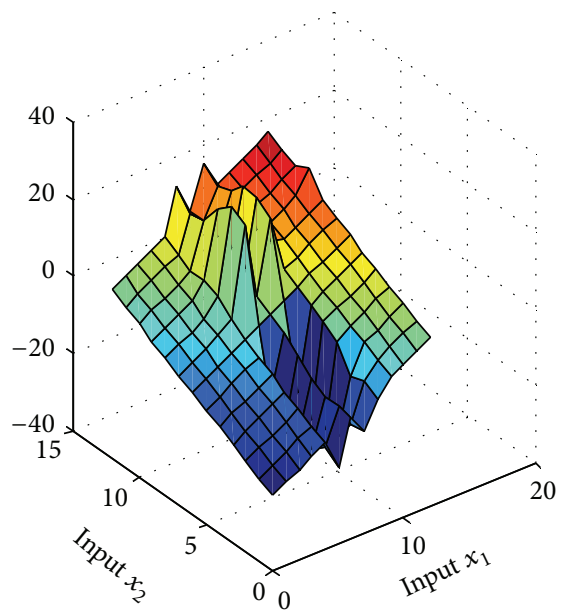

Number of sampled data: 4500

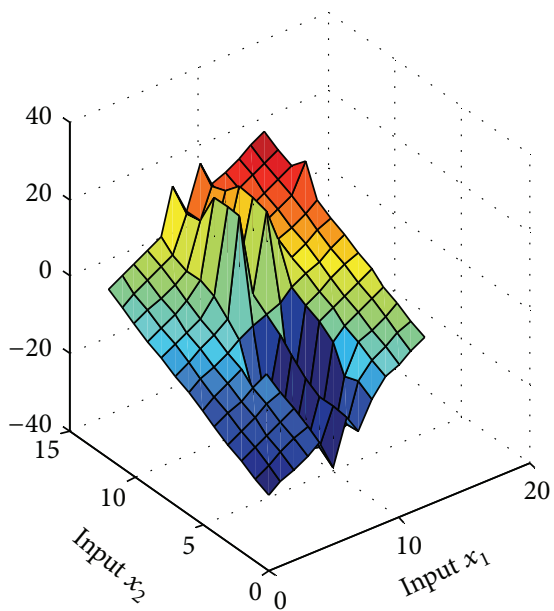

FIGURE 9: Training result of predesigned fuzzy surface.
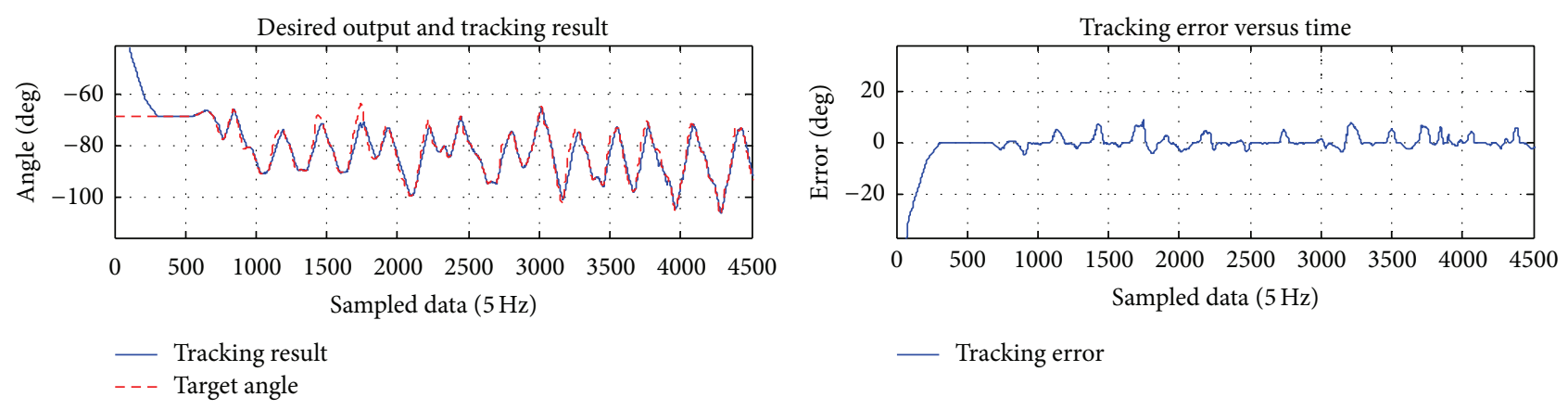

FIGURE 10: Tracking error of random set fuzzy surface with proposed ANFIS. 

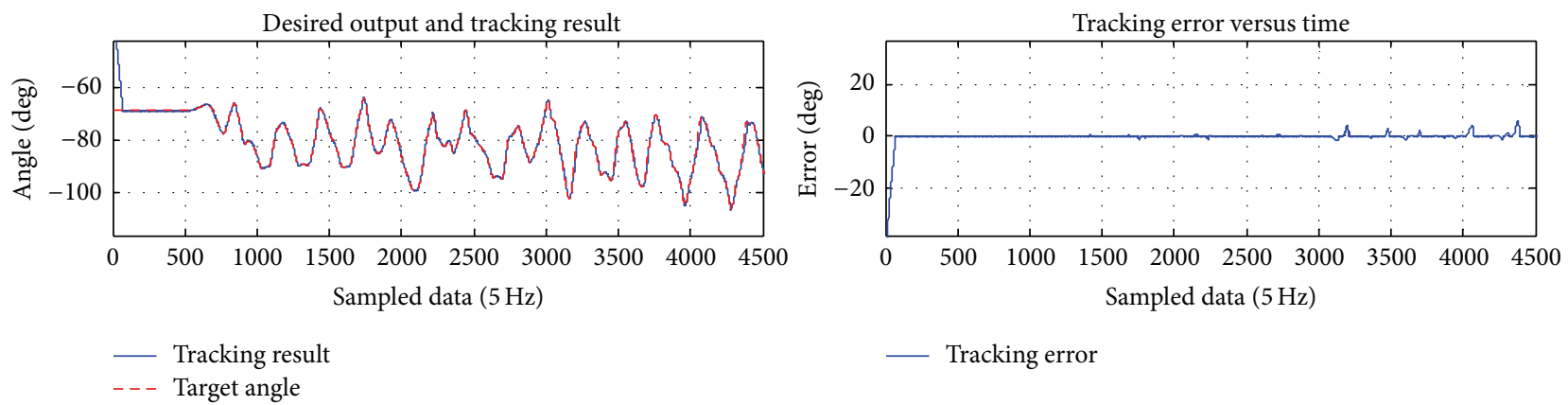

FIGURE 11: Tracking error of predesigned fuzzy surface with proposed ANFIS.

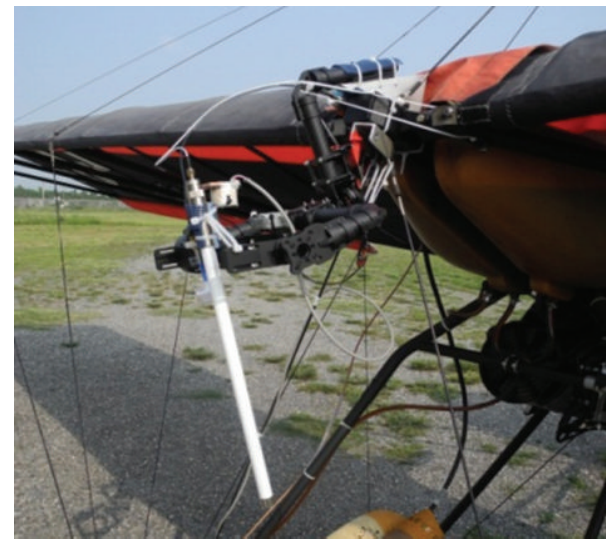

FIGURE 12: Airborne antenna on JJ2071 for ULAFP test.

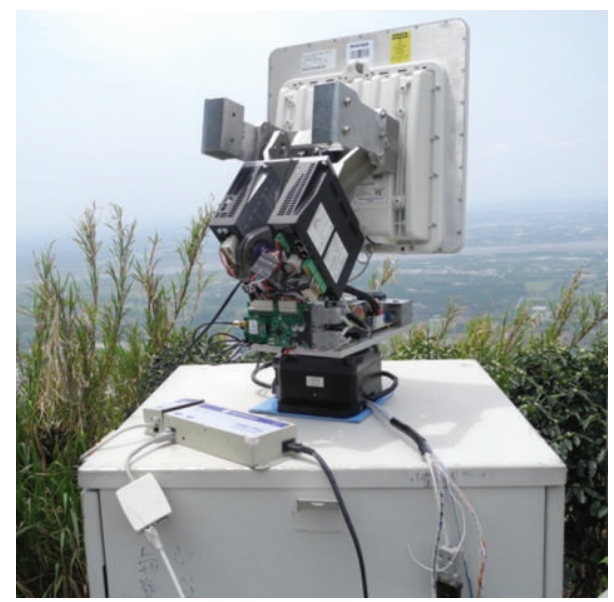

FIGURE 13: Ground antenna tracking mechanism with microwave module.

Ground antenna tracking mechanism is set up at mountainside in southern Taiwan, as shown in Figure 13, at above ground level (AGL) $334 \mathrm{~m}$ (or about $440 \mathrm{~m} \mathrm{MSL}$ ). A PTP58500 transceiver with integrated panel antenna is mounted at 2-axis rotation mechanism with well alignment on both azimuth and elevation axes. The test site is shown in Figure 14.

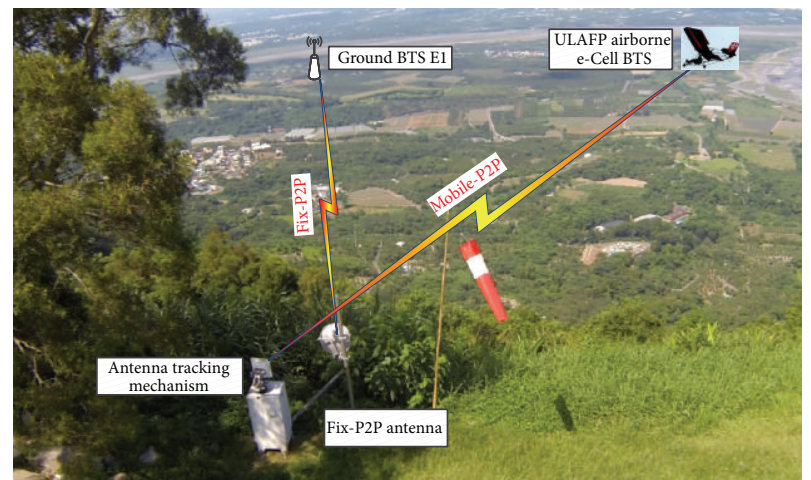

FIgure 14: Test site for Sky-Net microwave air-bridging at $440 \mathrm{~m}$ (MSL).

Flight test path was designed to ensure that both sides of microwave module antenna pattern can be overlapped. In order to verify the tracking system being capable of maintaining constant microwave link, the fight paths and distances are predetermined to check the microwave link. The flying platform test altitude is fixed at $440 \mathrm{~m} \sim 450 \mathrm{~m}$ (MSL) as shown with flight path in Figure 15.

Telecom data package in backbone network of Chunghwa Telecom is transmitted in E1 format, and therefore MAB system has a bandwidth threshold $(3.5 \mathrm{Mbps})$ in order to satisfy the basic telecom data exchanging to establish a stable mobile communication service. Table 2 shows that both system signal strength and vector errors are factors which affect system maximum throughput. As the wireless link status is changed due to antenna misalignment or weather condition, microwave module would change the modulation in order to fit current wireless link condition. Therefore, the sticking point of maintaining the QoS of MAB falls on keeping the system signal strength higher than $-87.96 \mathrm{dBm}$ and vector error is lower than $-11 \mathrm{~dB}$, shown by red line in Figures 16 and 17. These are also the threshold to hold the QPSK 0.75 modulation to provide a steady $3.54 \mathrm{Mbps}$ system bandwidth.

The tracking steps are classified into take-off, test phase in cruise, and landing. In order to ensure that the system signal strength scale is qualified for $\mathrm{MAB}$, the ground tracking unit must maintain ULAFP under area coverage of conical 

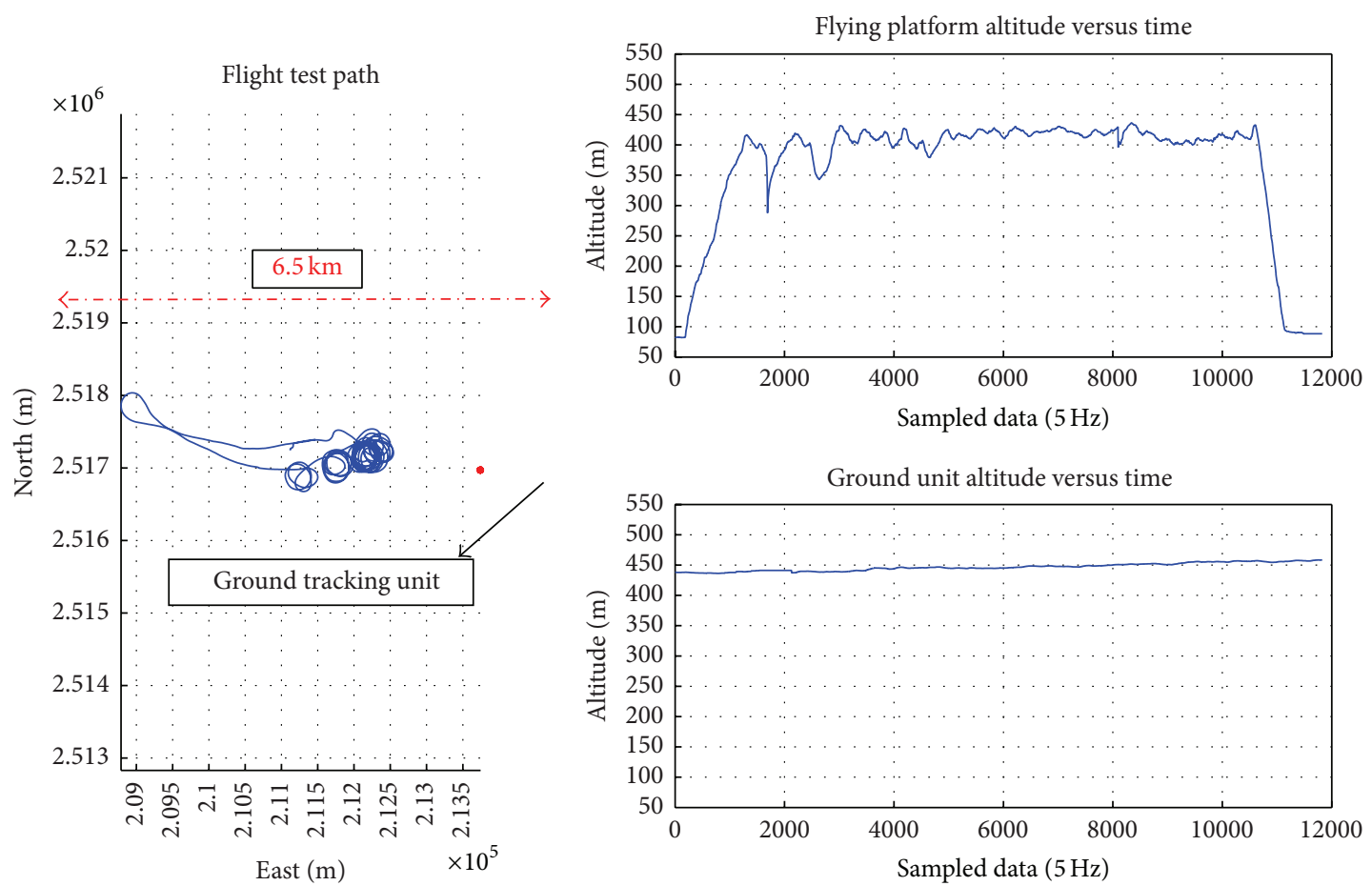

FIGURE 15: Flight test path and altitude.

TABLE 2: PTP58500 adaptive system modulation.

\begin{tabular}{lccc}
\hline Modulation mode & $\begin{array}{c}\text { Signal } \\
\text { threshold } \\
(\mathrm{dBm})\end{array}$ & $\begin{array}{c}\text { Vector error } \\
(\mathrm{dB})\end{array}$ & $\begin{array}{c}\text { Bandwidth } \\
(\mathrm{Mbps})\end{array}$ \\
\hline 16QAM 0.75 & -81 & -17.8 & 7.39 \\
16QAM 0.50 & -84.78 & -14.5 & 5.45 \\
QPSK 0.75 & $-\mathbf{8 7 . 9 6}$ & $-\mathbf{1 1}$ & $\mathbf{3 . 5 4}$ \\
QPSK 0.50 & -89.78 & -9.5 & 2.04 \\
BPSK 0.50 & -99.02 & -1.9 & 0.61 \\
\hline
\end{tabular}

TABLE 3: Antenna/transceiver specification of ground tracking unit.

\begin{tabular}{lc}
\hline Antenna polarization & Linear \\
\hline H-HPBW/V-HPBW & 8 degrees \\
\hline Gain & $23 \mathrm{dBi}$ \\
\hline Transceiver Tx power & $-18 \sim 27 \mathrm{dBm}$ \\
\hline Transceiver sensitivity & $-69 \sim-94 \mathrm{dBm}$ \\
\hline
\end{tabular}

antenna pattern. According to the ground directional panel antenna pattern and microwave module specification in Table 3, the ground tracking mechanism is designed with predetermined flight path as Table 4 to match with the ideal MAB performance.

The preliminary flight test results with flight path in Table 3 are shown in Figures 16 and 17, for tracking mechanism using random set and predesigned fuzzy surface with the proposed ANFIS method.

Since Half-Power Beam Width (HPBW) of the directional panel antenna on the ground has 8 degrees for both vertical
TABLE 4: Ground tracking mechanism specification and flying path.

\begin{tabular}{|c|c|}
\hline \multicolumn{2}{|c|}{ Flying platform } \\
\hline Cruising speed & $60 \sim 72 \mathrm{~km} / \mathrm{hr}$ \\
\hline Flight path distance & $2.5 \sim 6 \mathrm{~km}$ \\
\hline Turning radius & $500 \mathrm{~m}$ \\
\hline \multicolumn{2}{|c|}{ Antenna tracking mechanism } \\
\hline $\begin{array}{l}\text { Azimuth/elevation rotary } \\
\text { angle }\end{array}$ & 360 degrees/-15 90 degrees \\
\hline $\begin{array}{l}\text { Azimuth/elevation tracking } \\
\text { speed }\end{array}$ & $10 \mathrm{dps} / 5 \mathrm{dps}^{*}$ (max.) \\
\hline
\end{tabular}

*dps: degree per second.

and horizontal patterns, $\mathrm{MAB}$ is very sensitive on antenna alignment accuracy. Even a small tracking delay could lead the system bandwidth to drop drastically or be disconnected. The gap in Figure 15 is caused by control core "step" into the logic confliction region while tracking, in which rule is formed by random set fuzzy surface as neural networks just starting the training process. Even though the fuzzy surface would be trained appropriately for flight mission as time goes on, there are still some regions that never get updated which hide the potential output risk for the whole tracking system. On the other hand, the proposed rule formation methods with predesigned fuzzy surface have capability of being less time consuming on rule training and maintain coherent output result. This sustains MAB throughput for $4 \mathrm{Mbps}$ more than the determined bandwidth threshold. This is shown in Figure 17. The steady MAB throughput, as shown in Figure 17, is therefore qualified for relaying telecom signal 

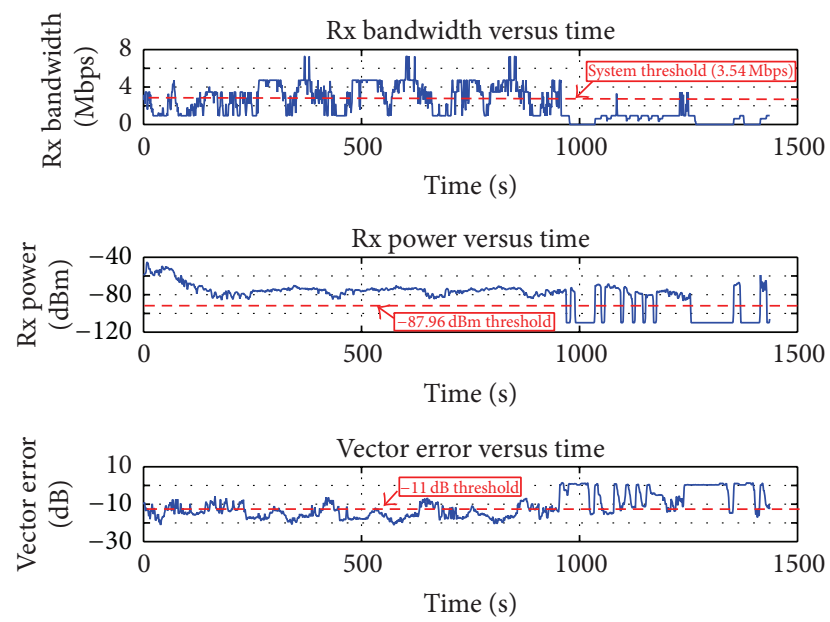

FIgURE 16: System bandwidth, Rx power, and vector error of MAB (random set fuzzy surface).
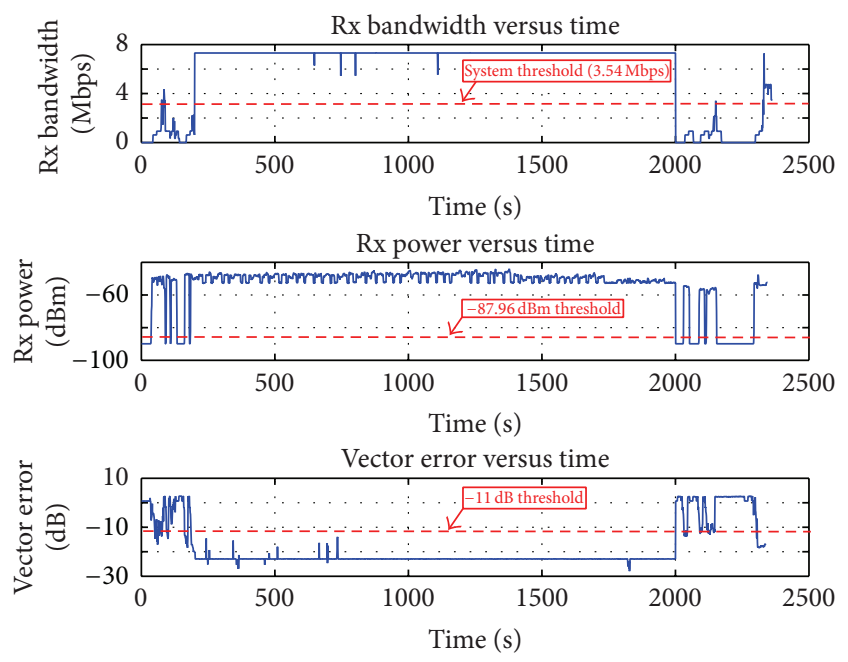

Figure 17: System bandwidth, Rx power, and vector error of MAB (predesigned fuzzy surface).

with $\mathrm{E} 1$ format. This is the key technique support for further applications with airborne $e$-Cell BTS in the Sky-Net project.

\section{Conclusion}

The Sky-Net project proposes a temporary airborne mobile communication service in disaster stroke area. A ULAFP is used to carry e-Cell BTS to relay mobile service via microwave transceiver for general users to the backbone network of the mobile communication providers. With the demand on high throughput and steady microwave wireless link, directional antenna is adopted for ground tracking device to establish reliable $\mathrm{MAB}$ between flying platform and ground E1. It is designed for aiming airborne antenna on ULAFP with circular and linear polarization, separately. In consideration of maintaining airborne antenna polarization during flight missions, an airborne self-stabilized gimbal is introduced for ensuring the attitude of antennas regardless of ULAFP's dynamic motion. Meanwhile, performance of ground antenna tracking mechanism is planned to establish microwave link adapting different tracking scenario and ensure the control output being coherent. Therefore, an ANFIS with modified rule formation layer is implemented into the control core. Under the proposed control method, tracking result can maintain logical coherence and achieve accurate directional antenna alignment to mobile target. Both airborne stabilization and ground antenna tracking mechanism performance are verified via flight test in this paper. Test results have strongly been proven with the receiving bandwidth and power for MAB in high quality and good integrity by link status data log. This is the evidence to support the proposed Sky-Net architecture being feasible and effective for relaying telecommunication signal through ULAFP from the ground infrastructure of mobile system provider into designed areas.

\section{Conflict of Interests}

The authors declare that there is no conflict of interests regarding the publication of this paper.

\section{Acknowledgment}

This work is supported by National Science Council for SkyNet Development under Contract NSC 102-2221-E-006-081MY3.

\section{References}

[1] A. K. Widiawan and R. Tafazolli, "High Altitude Platform Station (HAPS): a review of new infrastructure development for future wireless communications," Wireless Personal Communications, vol. 42, no. 3, pp. 387-404, 2007.

[2] Q. Feng, J. McGeehan, and A. R. Nix, "Enhancing coverage and reducing power consumption in peer-to-peer networks through airborne relaying," in Proceedings of the IEEE 65th Vehicular Technology Conference (VTC-Spring '07), pp. 954-958, April 2007.

[3] D. Grace, J. Thornton, G. Chen, G. P. White, and T. C. Tozer, "Improving the system capacity of broadband services using multiple high-altitude platforms," IEEE Transactions on Wireless Communications, vol. 4, no. 2, pp. 700-709, 2005.

[4] Z. E. O. Elshaikh, M. R. Islam, A. F. Ismail, and O. O. Khalifa, "High altitude platform for wireless communications and other services," in Proceedings of the 4th International Conference on Electrical and Computer Engineering (ICECE '06), pp. 432-438, December 2006.

[5] H. Hariyanto, H. Santoso, and A. K. Widiawan, "Emergency broadband access network using low altitude platform," in Proceedings of the International Conference on Instrumentation, Communication, Information Technology, and Biomedical Engineering (ICICI-BME '09), pp. 1-6, November 2009.

[6] A. Qiantori, A. B. Sutiono, H. Hariyanto, H. Suwa, and T. Ohta, "An emergency medical communications system by low altitude platform at the early stages of a natural disaster in Indonesia," Journal of Medical Systems, vol. 36, no. 1, pp. 41-52, 2012. 
[7] W. Guo, C. Devine, and S. Wang, "Performance analysis of micro unmanned airborne communication relays for cellular networks," in Proceedings of the 9th International Symposium on Communication Systems, Networks \& Digital Signal Processing (CSNDSP '14), pp. 658-663, IEEE, Manchester, UK, July 2014.

[8] D. Grace, J. Thornton, C. Spillard, T. Konefal, and T. C. Tozer, "Broadband communications from a high-altitude platform: the european helinet programme," Electronics and Communication Engineering Journal, vol. 13, no. 3, pp. 138-144, 2001.

[9] E. Falletti, M. Laddomada, M. Mondin, and F. Sellone, "Integrated services from high-altitude platforms: a flexible communication system," IEEE Communications Magazine, vol. 44, no. 2, pp. 85-94, 2006.

[10] R. Miura and M. Suzuki, "Preliminary flight test program on telecom and broadcasting using high altitude platform stations," Wireless Personal Communications, vol. 24, no. 2, pp. 341-361, 2003.

[11] M. Quigley, M. A. Goodrich, S. Griffiths, A. Eldredge, and R. W. Beard, "Target acquisition, localization, and surveillance using a fixed-wing mini-UAV and gimbaled camera," in Proceedings of the IEEE International Conference on Robotics and Automation (ICRA '05), pp. 2600-2606, April 2005.

[12] M. M. Abdo, A. R. Vali, A. R. Toloei, and M. R. Arvan, "Stabilization loop of a two axes gimbal system using self-tuning PID type fuzzy controller," ISA Transactions, vol. 53, no. 2, pp. 591-602, 2014.

[13] S. O. H. Madgwick, A. J. L. Harrison, and R. Vaidyanathan, "Estimation of IMU and MARG orientation using a gradient descent algorithm," in Proceedings of the IEEE International Conference on Rehabilitation Robotics (ICORR '11), pp. 1-7, July 2011.

[14] T. Takagi and M. Sugeno, "Fuzzy identification of systems and its applications to modeling and control," IEEE Transactions on Systems, Man and Cybernetics, vol. 15, no. 1, pp. 116-132, 1985.

[15] C. E. Lin, T.-Y. Lu, H.-Y. Chen, and Y.-H. Lai, "System reengineering in mechanism design and implementation for unmanned ultra-light (part I)," Journal of Aeronautics, Astronautics and Aviation Series A, vol. 46, no. 2, pp. 124-131, 2014.

[16] C. E. Lin, H.-Y. Chen, T.-Y. Lu, and Y.-C. Huang, "System reengineering in flight control verification for unmanned ultralight (part II)," Journal of Aeronautics, Astronautics and Aviation Series A, vol. 46, no. 2, pp. 132-140, 2014. 

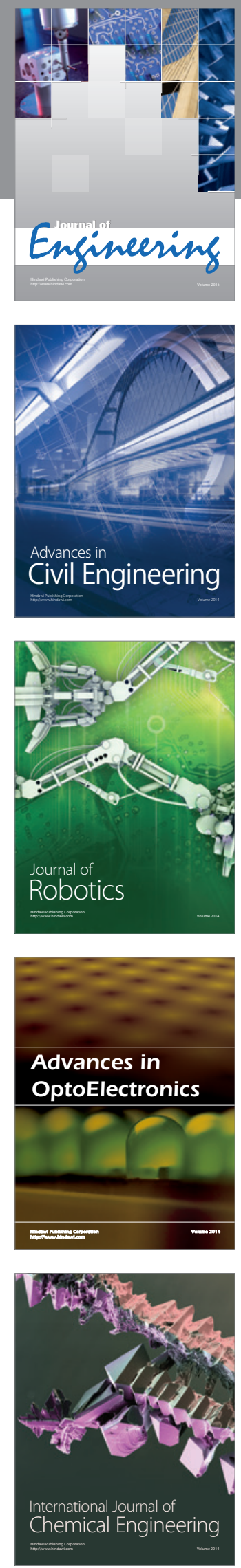

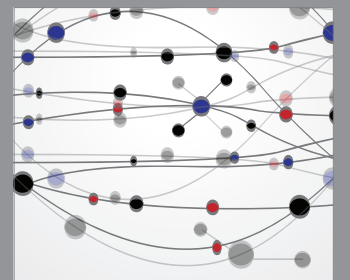

The Scientific World Journal
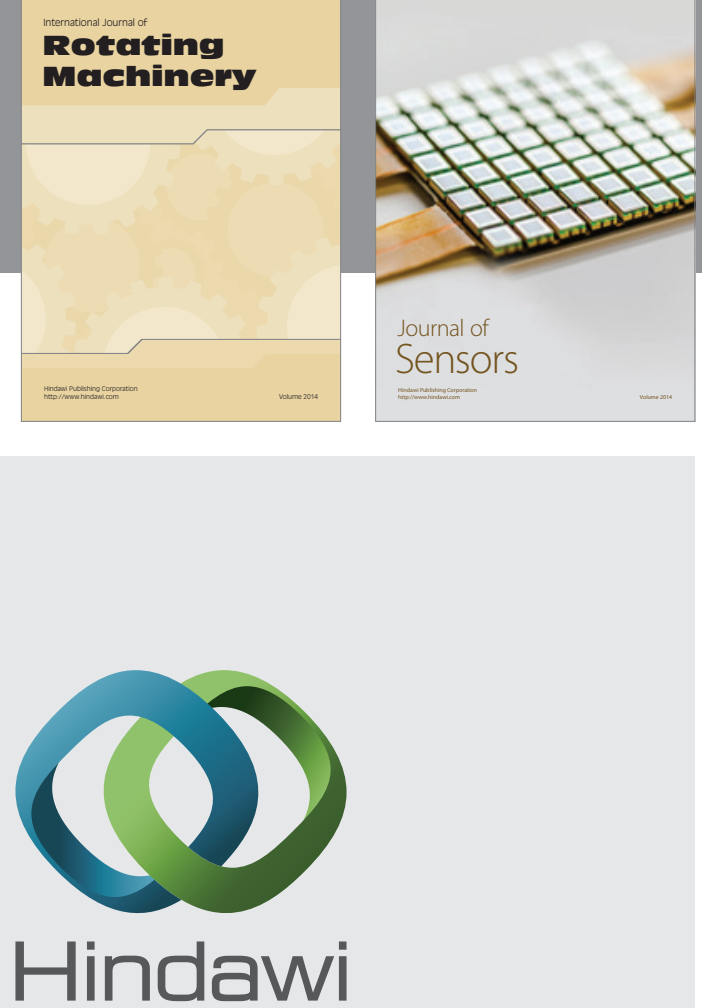

Submit your manuscripts at http://www.hindawi.com
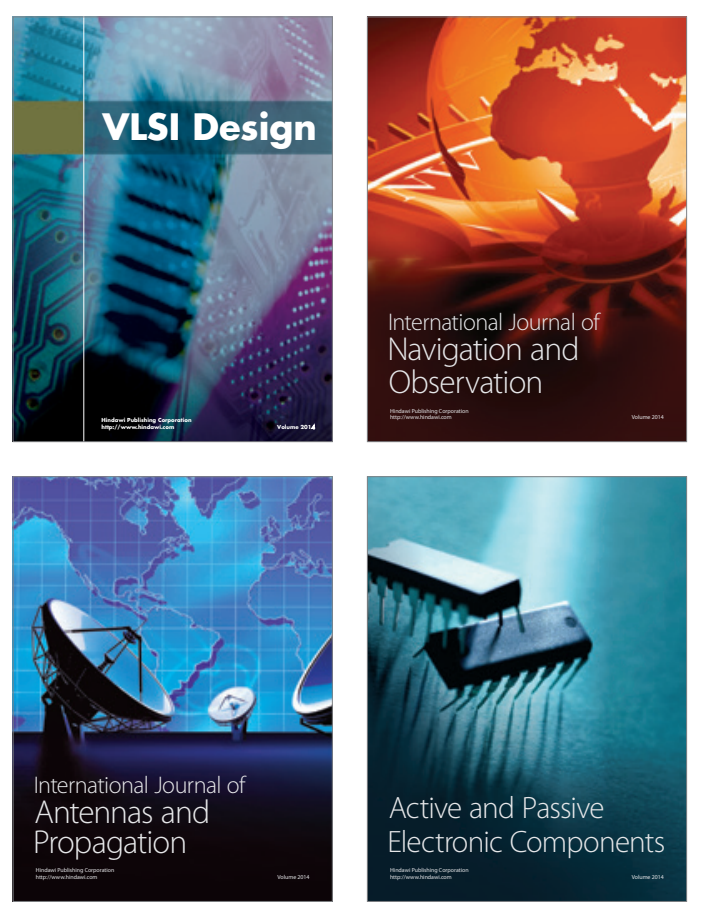
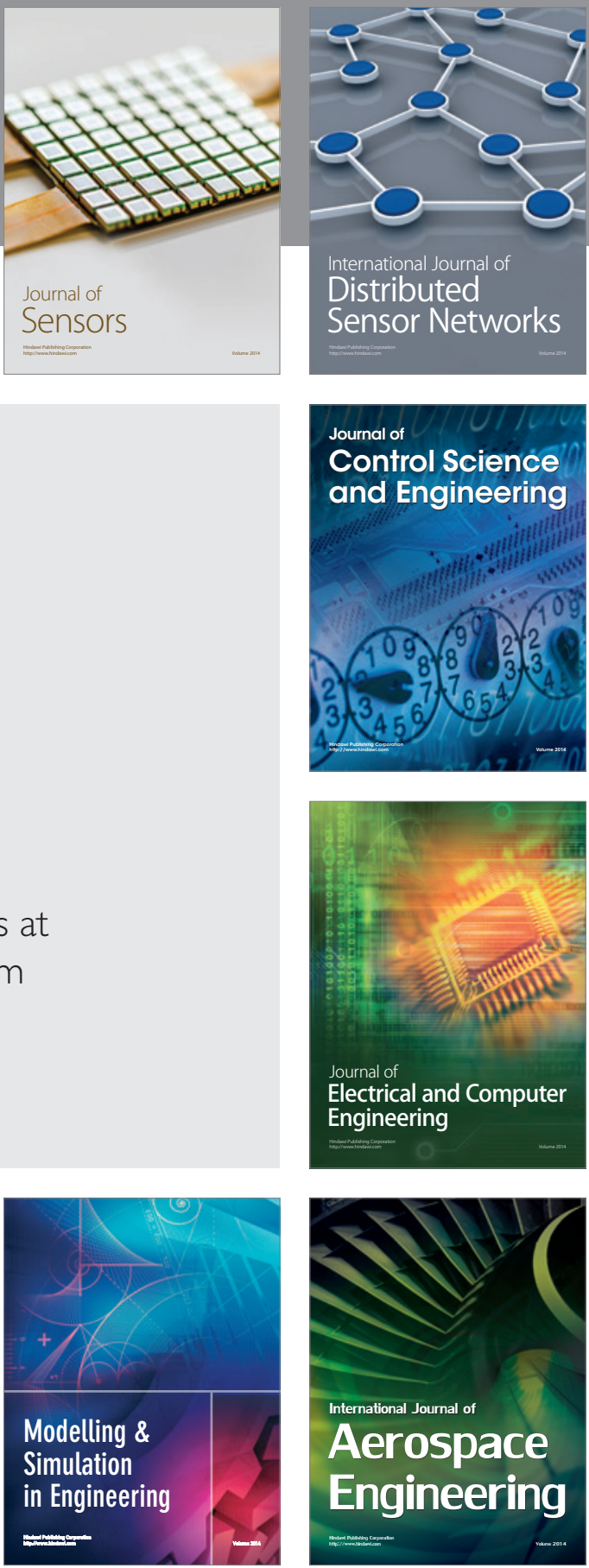

Journal of

Control Science

and Engineering
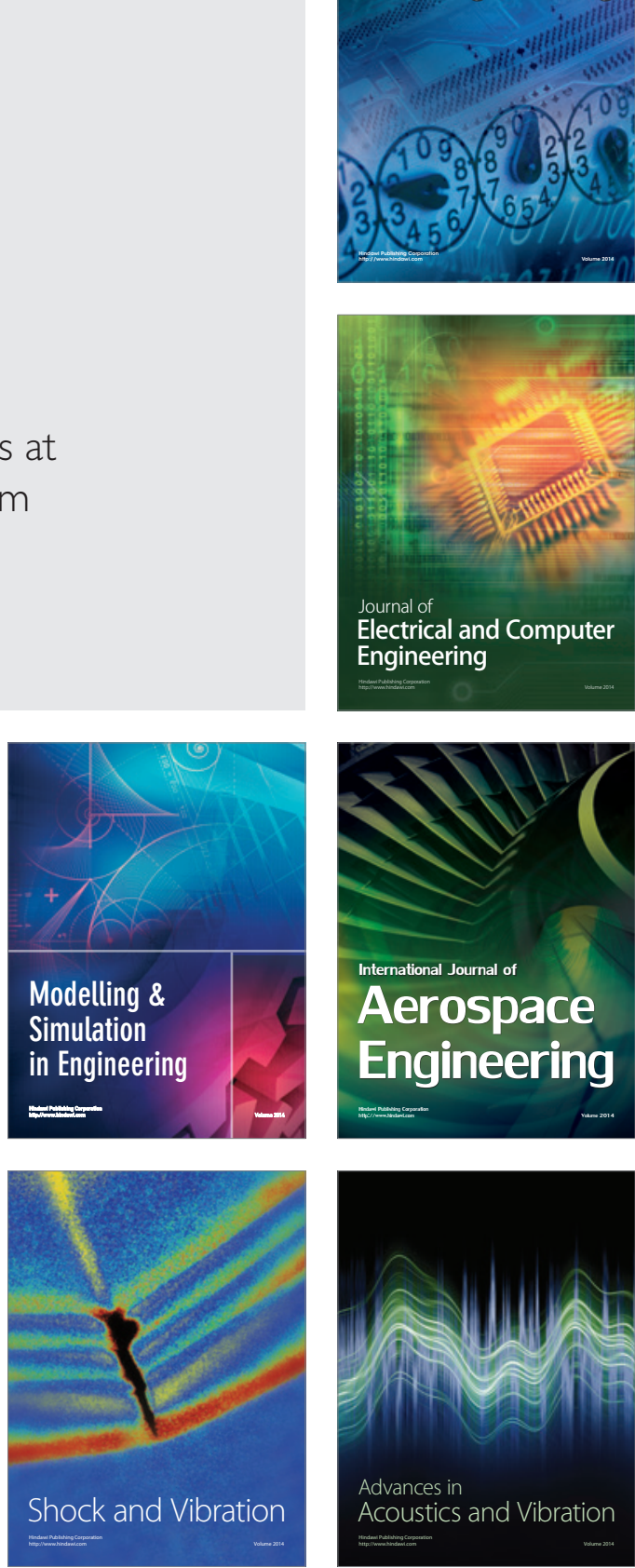